\title{
ON THE INDEX OF A FIBERED MANIFOLD ${ }^{1}$
}

\section{S. S. CHERN, F. HIRZEBRUCH, AND J-P. SERRE}

Introduction. Let $V$ be a real vector space of dimension $r$. Let $F(x, y)=\langle x, y\rangle, x, y \in V$, be a real-valued symmetric bilinear function. We can find a base $e_{i}, 1 \leqq i \leqq r$, in $V$, such that

$$
F(x, y)=\sum_{i=1}^{p} x^{i} y^{i}-\sum_{i=p+1}^{p+q} x^{i} y^{i}
$$

where $x=\sum_{i=1}^{r} x^{i} e_{i}$ and $y=\sum_{i=1}^{r} y^{i} e_{i}$.

The number $p-q$ is called the index of $F$, to be denoted by $\tau(F)$. It depends only on $F$. If $F$ is nonsingular (i.e. $p+q=r$ ), then $\min (p, q)$ equals the maximal dimension of the linear subspaces of $V$ contained in the "cone" $F(x, x)=0$.

Now let $M$ be a compact oriented manifold. The index of $M$ is defined to be zero, if the dimension of $M$ is not a multiple of 4 . If $M$ has the dimension $4 k$, consider the cohomology group $H^{2 k}(M)$ with real coefficients. This is a real vector space, and the equation

$$
\langle x, y\rangle \xi=x \cup y, \quad x, y \in H^{2 k}(M),
$$

where $\xi$ is the generator of $H^{4 k}(M)$ defined by the given orientation of $M$, defines a real-valued symmetric bilinear form $\langle x, y\rangle$ over $H^{2 k}(M)$. Its index is called the index of $M$, to be denoted by $\tau(M)$. Reversal of the orientation of $M$ changes the sign of the index. The form $\langle x, y\rangle$ defined by (2) is nonsingular, since, by Poincaré's duality theorem, the equation $x \cup y=0$ for all $x \in H^{2 k}(M)$ implies $y=0$.

The main purpose of this paper is to prove the theorem:

THEOREM. Let $E \rightarrow B$ be a fiber bundle, with the typical fiber $F$, such that the following conditions are satisfied:

(1) $E, B, F$ are compact connected oriented manifolds;

(2) The fundamental group $\pi_{1}(B)$ acts trivially on the cohomology ring $H^{*}(F)$ of $F$.

Then, if $E, B, F$ are oriented coherently, so that the orientation of $E$ is induced by those of $F$ and $B$, the index of $E$ is the product of the indices of $F$ and $B$, that is,

$$
\tau(E)=\tau(F) \tau(B)
$$

Received by the editors September 7, 1956.

1 Work done when the first named author was under partial support by the National Science Foundation. 
REMARK. We do not know whether condition (2) and the connectedness hypothesis of condition (1) are necessary. For instance, let $E$ be an $n$-sheeted covering of $B$ (the spaces $B$ and $E$ still being compact oriented manifolds); is it true that $\tau(E)=n \tau(B)$ ? We know the answer to be positive only when $B$ possesses a differentiable structure: in that case, according to a theorem of one of us, $\tau(B)$ (resp. $\tau(E)$ ) is equal to the Pontrjagin number $L(B)$ (resp. $L(E)$ ) and it is clear that $L(E)=n \cdot L(B)$.

1. Algebraic properties of the index of a matrix. Let $e_{i}, 1 \leqq i \leqq r$, be a base in $V$. A real-valued symmetric bilinear function $\langle x, y\rangle$ defines a real-valued symmetric matrix $C=\left(c_{i j}\right), c_{i j}=\left\langle e_{i}, e_{j}\right\rangle, 1 \leqq i, j \leqq r$, and is determined by it. The index of the bilinear function is equal to the index $\tau(C)$ of $C$, if we define the latter to be the excess of the number of positive eigenvalues over the number of negative eigenvalues of $C$, each counted with its proper multiplicity. We have the following properties of the index of a real symmetric matrix:

For a nonsingular $(r \times r)$-matrix $T$ we have

$$
\tau(C)=\tau\left({ }^{t} T C T\right) .
$$

Here, as always, we denote by ${ }^{t} T$ the transpose of $T$. For nonsingular square matrices $A, L$ (with $A$ symmetric) we have

$$
\tau\left(\begin{array}{ccc}
0 & 0 & L \\
0 & A & 0 \\
{ }^{t} L & 0 & 0
\end{array}\right)=\tau\left(\begin{array}{cc}
0 & L \\
{ }{ }^{L} L & 0
\end{array}\right)+\tau(A)=\tau(A) .
$$

Here and always we make use of the convention that the index of the empty matrix is zero.

To prove (4) it is enough to show that

$$
\tau\left(\begin{array}{cc}
0 & L \\
{ }^{t} L & 0
\end{array}\right)=0
$$

In this case, $r$ is even. Put $r=2 \mu$. Obviously, the cone $F(x, x)=0$ of the symmetric bilinear function $F(x, y)$ belonging to the matrix

$$
\left(\begin{array}{cc}
0 & L \\
t L & 0
\end{array}\right)
$$

contains a linear space of dimension $\mu$. Thus $\min (p, q) \geqq \mu$. On the other hand, $p+q=2 \mu$. Therefore, $p=q$ and $\tau=0$.

LEMMA 1. Let $C$ be a real, symmetric, nonsingular matrix of the form 


$$
C=\left(\begin{array}{ccc}
0 & & L_{0} \\
& \cdot & \\
L_{m} & & *
\end{array}\right)
$$

where $L_{0}, \cdots, L_{m}$ are square matrices (empty matrices are admitted) and where $L_{i}$ is the transpose of $L_{m-i}$. Then

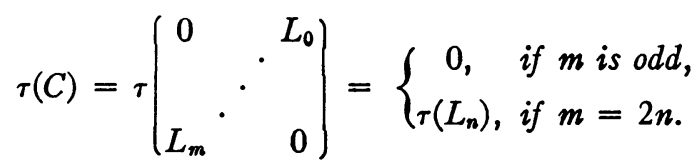

Proof. We put

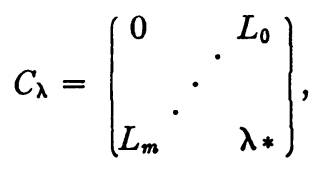

Since $\operatorname{det}\left(C_{\lambda}\right)= \pm \prod_{i=0}^{m} \operatorname{det}\left(L_{i}\right) \neq 0$, the index $\tau\left(C_{\lambda}\right)$ is obviously independent of $\lambda$, so that $\tau(C)=\tau\left(C_{1}\right)=\tau\left(C_{0}\right)$. By (4) we have $\tau\left(C_{0}\right)=0$ resp. $\tau\left(C_{0}\right)=\tau\left(L_{n}\right)$, q.e.d.

Lemma 2. Let $A$ and $B$ be two square matrices, which are either both symmetric or both skew-symmetric. Then their tensor product $A \otimes B$ is symmetric, and

$$
\tau(A \otimes B)=\tau(A) \tau(B) \text { or } 0,
$$

according as both $A$ and $B$ are symmetric or skew-symmetric.

Suppose first that $A$ and $B$ are both symmetric. Let $\alpha_{i}>0, \alpha_{j}<0$, $1 \leqq i \leqq p, p+1 \leqq j \leqq p+q$, be the nonzero eigenvalues of $A$ and $\beta_{k}>0$, $\beta_{l}<0,1 \leqq k \leqq p^{\prime}, p^{\prime}+1 \leqq l \leqq p^{\prime}+q^{\prime}$ be the nonzero eigenvalues of $B$. Then the nonzero eigenvalues of $A \otimes B$ are $\alpha_{u} \beta_{t}, 1 \leqq u \leqq p+q$, $1 \leqq t \leqq p^{\prime}+q^{\prime}$. It follows that

$$
\tau(A \otimes B)=p p^{\prime}+q q^{\prime}-p q^{\prime}-p^{\prime} q=\tau(A) \tau(B) .
$$

Now let $A$ and $B$ be both skew-symmetric. By applying (3) to the matrix $C=A \otimes B$ we can suppose that $A$ and $B$ are both of the form

$$
\left(\begin{array}{llll}
A_{1} & & & 0 \\
& \ddots & & \\
& & A_{n} & \\
0 & & & 0
\end{array}\right)
$$


where each $A_{i}$ is a $2 \times 2$ block:

$$
A_{i}=\left(\begin{array}{rr}
0 & 1 \\
-1 & 0
\end{array}\right)=J
$$

Since

$$
\tau\left(\left(\begin{array}{rr}
0 & 1 \\
-1 & 0
\end{array}\right) \otimes\left(\begin{array}{rr}
0 & 1 \\
-1 & 0
\end{array}\right)\right)=\tau\left(\begin{array}{rr}
0 & J \\
t J & 0
\end{array}\right)=0,
$$

we have $\tau(A \otimes B)=0$.

2. Poincare rings. We consider a graded ring $A$ with the following properties:

(1) In the direct sum decomposition

$$
A=\sum_{0 \leq r<\infty} A^{r}
$$

of $A$ into the subgroups of its homogeneous elements, each $A^{r}$ is a real vector space of finite dimension. There exists an $n$ with $A^{r}=0$ for $r>n$ and with $\operatorname{dim} A^{n}=1$.

(2) If $x \in A^{i}, y \in A^{i}$ then $x y \in A^{i+j}$ and

$$
x y=(-1)^{i j} y x \text {. }
$$

Let $\xi \neq 0$ be a base element of $A^{n}$. Relative to $\xi$ we define a bilinear pairing $\langle x, y\rangle$ of $A^{r}$ and $A^{n-r}$ into the real field by the equation

$$
\langle x, y\rangle \xi=x y, \quad x \in A^{r}, y \in A^{n-r} .
$$

Let $i_{n-r}$ be the linear mapping of $A^{n-r}$ into $\left(A^{r}\right)^{*}$, the dual vector space of $A^{r}$, which assigns to $y \in A^{n-r}$ the linear function $\langle x, y\rangle$ on $A^{r}\left(x \in A^{r}\right)$.

A graded ring $A$ is called a Poincaré ring if it satisfies (1), (2) and has moreover the following property:

(3) The mapping $i_{n-r}$ is a bijection of $A^{n-r}$ onto $\left(A^{r}\right)^{*}$.

A consequence of $(3)$ is

$$
\operatorname{dim} A^{r}=\operatorname{dim} A^{n-r}, \quad 0 \leqq r \leqq n .
$$

The cohomology ring of a compact orientable manifold is a Poincaré ring.

A differentiation in a Poincaré ring $A$ is a linear endomorphism $d: A \rightarrow A$, satisfying the following conditions:
( $\alpha) d A^{r} \subset A^{r+1}$;
(B) $d d=0$;
( $\gamma) d(x y)=(d x) y+(-1)^{r} x(d y)$, if $x \in A^{r}$;
(ס) $d A^{n-1}=0$. 
As is well known, such a differentiation defines a derived ring $A^{\prime}=d^{-1}(0) / d A$. If we put $A^{\prime r}=d^{-1}(0) \cap A^{r} / d A^{r-1}$, we have the direct sum decomposition

$$
A^{\prime}=\sum_{0 \leq r \leq n} A^{\prime r},
$$

and $A^{\prime}$ is a graded ring. It is easy to verify that, if $x^{\prime} \in A^{\prime i}, y^{\prime} \in A^{\prime i}$, then $x^{\prime} y^{\prime} \in A^{\prime i+j}$, and

$$
x^{\prime} y^{\prime}=(-1)^{i j} y^{\prime} x^{\prime} .
$$

From the property $(\delta)$ of $d$ we have $\operatorname{dim} A^{\prime n}=1$. Thus $A^{\prime}$ satisfies (1) and (2) with the same maximal degree $n$ as $A$. We denote the residue class of $\xi$ in $A^{\prime n}$ by $\xi^{\prime}$. Relative to $\xi^{\prime}$ we have the linear mapping

$$
i_{n-r}^{\prime}: A^{\prime n-r} \rightarrow\left(A^{\prime r}\right)^{*} \text {. }
$$

Lemma 3. The derived ring of a Poincare ring with differentiation is a Poincare ring, i.e. $i_{n-r}^{\prime}$ is bijective.

It remains to prove that $A^{\prime}$ has the property (3) in the definition of a Poincaré ring. Let $x \in A^{r}, y \in A^{n-r-1}$. By property $(\delta)$ of $d$, we have

$$
0=d(x y)=(d x) y+(-1)^{r} x(d y) \text {. }
$$

This gives

$$
\langle d x, y\rangle=(-1)^{r-1}\langle x, d y\rangle,
$$

a relation which is independent of the choice of $\xi$. This relation is equivalent to saying that the following diagram is commutative:

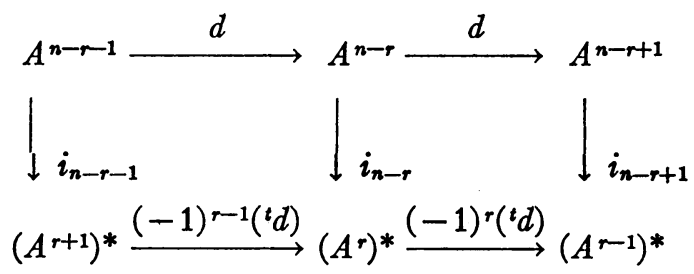

where $\left(A^{r}\right)^{*}$ is the dual space of $A^{r}$, and ${ }^{t} d$ is the dual homomorphism of $d$. We have the canonical isomorphism

$$
\left(A^{\prime r}\right)^{*} \cong{ }^{t} d^{-1}(0) \cap\left(A^{r}\right)^{*} /{ }^{t} d\left(A^{r+1}\right)^{*} .
$$

The above diagram shows that $i_{n-r}$ induces an isomorphism, namely $i_{n-r}^{\prime}$, of $A^{\prime n-r}$ onto $\left(A^{\prime r}\right)^{*}$. It follows that $A^{\prime r}$ and $A^{\prime n-r}$ are dually paired into the real field relative to the element $\xi^{\prime} \in A^{\prime n}$, which is the residue class of $\xi$. 
In analogy with the index of an oriented manifold we can define the index $\tau_{\xi}(A)$ of our Poincaré ring $A$ relative to $\xi$. It is to be zero, if $n \equiv 0, \bmod 4$. If $n=4 k, \tau_{\xi}(A)$ is to be the index of the bilinear function $\langle x, y\rangle, x, y \in A^{2 k}$. Obviously, $\tau_{\xi}(A)=\tau_{\xi_{1}}(A)$, if $\xi_{1}$ is a positive multiple of $\xi$.

Lemma 4. In a Poincaré ring $A$ let $\xi \neq 0$ be a base of $A^{n}$, and let $\xi^{\prime} \in A^{\prime n}$ be the residue class which contains $\xi$. Then $\tau_{\xi^{\prime}}\left(A^{\prime}\right)=\tau_{\xi}(A)$.

It is only necessary to prove the lemma for the case $n=4 k$. Let $Z^{2 k}=d^{-1}(0) \cap A^{2 k}, B^{2 k}=d A^{2 k-1}$, and let $a, b, c$ be the respective dimensions of $A^{2 k}, B^{2 k}, Z^{2 k}$. It follows immediately from (8) that each of the two spaces $B^{2 k}$ and $Z^{2 k}$ is the orthogonal of the other with respect to the symmetric form $\langle x, y\rangle$ of $A^{2 k}$, whence $a=b+c$. We have $B^{2 k} \subset Z^{2 k} \subset A^{2 k}$. If $e_{i}$ is a base of $A^{2 k}$ such that $e_{i} \in B^{2 k}$ for $1 \leqq i \leqq b$ and $e_{i} \in Z^{2 k}$ for $b+1 \leqq i \leqq c$, the matrix $\left(\left\langle e_{i}, e_{j}\right\rangle\right)$ has then the form

$$
\left(\begin{array}{ccc}
0 & 0 & L \\
0 & Q & * \\
{ }^{\prime} L & * & *
\end{array}\right),
$$

where $L$ and $Q$ are square nonsingular matrices, of orders $b$ and $c-b$ respectively. Its index is $\tau_{\xi}(A)$, while $\tau(Q)$ is $\tau_{\xi^{\prime}}\left(A^{\prime}\right)$. By Lemma 1 , we get therefore $\tau_{\xi^{\prime}}\left(A^{\prime}\right)=\tau_{\xi}(A)$, as contended.

3. Proof of the theorem. It suffices to prove the theorem (see Introduction) for the case $\operatorname{dim} E=4 k$, which we suppose from now on. We consider the cohomology spectral sequence $E_{r}^{p, q}, 2 \leqq r \leqq \infty$, of the bundle $E \rightarrow B$, with the real field as the coefficient field. Let

$$
E_{r}^{*}=\sum_{p+q=0} E_{r}^{p, q}, \quad E_{r}=\sum_{0 \leq s} E_{r}^{*}, \quad 2 \leqq r \leqq \infty .
$$

Each $E_{r}$ is a graded ring, satisfying $E_{r}^{s} E_{r}^{s^{\prime}} \subset E_{r}^{s+s^{\prime}}$ and also $E_{r}^{p, q} E_{r}^{p^{\prime}, q^{\prime}}$ $\subset E_{r}^{p+p^{\prime}, \varepsilon+\alpha^{\prime}}$. It has a differentiation $d_{r}$, such that $E_{r+1}$ is the derived ring of $E_{r}$. In our case $d_{r}$ is trivial for sufficiently large $r$ and $E_{\infty}$, or $E_{\mathrm{r}}$ for $r$ sufficiently large, is the graded ring belonging to a certain filtration of the cohomology ring of the manifold $E$. The term $E_{2}$ of the spectral sequence is by hypothesis (2) of our theorem isomorphic to $H^{*}\left(B, H^{*}(F)\right)=H^{*}(B) \otimes H^{*}(F)$, such that

$$
E_{2}^{p, q} \cong H^{p}\left(B, H^{q}(F)\right) \cong H^{p}(B) \otimes H^{q}(F) .
$$

If we identify $E_{2}^{p, q}$ with $H^{p}(B) \otimes H^{q}(F)$ under this isomorphism, the multiplication in $E_{2}$ is given by 


$$
\begin{gathered}
(b \otimes f)\left(b^{\prime} \otimes f^{\prime}\right)=(-1)^{p^{\prime} q}\left(b \cup b^{\prime}\right) \otimes\left(f \cup f^{\prime}\right), \\
b \in H^{p}(B), \quad b^{\prime} \in H^{p^{\prime}}(B), \quad f \in H^{q}(F), \quad f^{\prime} \in H^{q^{\prime}}(F) .
\end{gathered}
$$

Let $m=\operatorname{dim} F$, so that $\operatorname{dim} B=4 k-m$. Since $B$ and $F$ are manifolds, $E_{2}$ is a Poincaré ring with respect to the grading

$$
E_{2}=\sum_{0 \leq<<\infty} E_{2}^{*} \quad\left(E_{2}^{*}=0 \text { for } s>4 k, E_{2}^{4 k}=E_{2}^{4 k-m, m}\right) .
$$

The ring $E_{2}$ is isomorphic to the cohomology ring of $B \times F$.

The orientations of $B, F$ define a generator $\xi_{2}=\xi_{B} \otimes \xi_{F}$ of $E_{2}^{4 k}$. Here $\xi_{B}\left(\right.$ resp. $\xi_{F}$ ) denotes the generator of $H^{4 k-m}(B)$ (resp. $H^{m}(F)$ ) belonging to the orientation of $B$ (resp. $F$ ). We wish to prove that

We have

$$
\tau_{\xi_{2}}\left(E_{2}\right)=\tau(B) \cdot \tau(F) .
$$

$$
E_{2}^{2 k}=E_{2}^{2 k, 0}+E_{2}^{2 k-1,1}+\cdots+E_{2}^{2 k-m, m} .
$$

Here some of the $E_{2}^{p, a}$ might vanish, in particular $E_{2}^{p, q}=0$ if $p<0$. Clearly, for $x \in E_{2}^{2 k-a, q^{2}}$ and $y \in E_{2}^{2 k-q^{\prime}, q^{\prime}}$ we have $x y=0$ unless

$$
q+q^{\prime}=m \text {. }
$$

By Poincaré duality in $B$ and $F$, we have

$$
\operatorname{dim} E_{2}^{2 k-q, q}=\operatorname{dim} E_{2}^{2 k-m+q, m-q} .
$$

Therefore, the symmetric matrix, which defines the bilinear symmetric function over $E_{2}^{2 k}$, is, when written in blocks relative to the direct sum decomposition (9), of the form

$$
\left(\begin{array}{ccc}
0 & & L_{0} \\
& \cdot & \\
L_{m} & & 0
\end{array}\right)
$$

where the $L_{i}$ are nonsingular square matrices, such that $L_{\boldsymbol{i}}$ is the transpose of $L_{m-i}$. By Lemma 1 we obtain

$$
\tau_{\xi_{3}}\left(E_{2}\right)=0 \text { if } m \text { is odd, } \quad \tau_{\xi_{2}}\left(E_{2}\right)=\tau\left(L_{m / 2}\right) \text { if } m \text { is even. }
$$

In the first case the equation $\tau_{\xi_{3}}\left(E_{2}\right)=\tau(B) \tau(F)$ is proved, since $\tau_{\xi_{2}}\left(E_{2}\right)=\tau(F)=0$. In the latter case we have

$$
E_{2}^{2 k-m / 2, m / 2}=H^{2 k-m / 2}(B) \otimes H^{m / 2}(F),
$$


and it is clear that up to the sign $(-1)^{m / 2}$ the matrix $L_{m / 2}$ is the tensor product of the two matrices defining the bilinear forms of $B$ and $F$. If $\mathrm{m} / 2$ is odd, both matrices in this tensor product are skew-symmetric, and we have, by Lemma $2, \tau\left(L_{m / 2}\right)=0$; on the other hand we have $\tau(B) \tau(F)=0$, since $\operatorname{dim} F \not \equiv 0(\bmod 4)$ and thus by definition $\tau(F)=0$. If $m / 2$ is even, that is, if $m \equiv 0(\bmod 4)$, both matrices are symmetric, and Lemma 2 gives: $\tau\left(L_{m / 2}\right)=\tau(B) \tau(F)$. Combining all cases, we get the formula

$$
\tau_{\xi_{2}}\left(E_{2}\right)=\tau(B) \tau(F)
$$

in full generality.

The differentiation $d_{2}$ of $E_{2}$ satisfies the conditions of a differentiation in a Poincaré ring given in $\$ 2$. In fact, $\operatorname{dim} E_{\infty}^{\mathbf{k}}=1$, since $E$ is a manifold of dimension $4 k$. Therefore, $\operatorname{dim} E_{r}^{4 k}=1$ for $2 \leqq r$. Thus $d_{2}$ annihilates $E_{2}^{4 k-1}$; more generally $d_{r}$ annihilates $E_{r}^{4 k-1}$. It follows by Lemma 3 that $E_{3}$ is a Poincaré ring. It has $d_{3}$ as differentiation and therefore $E_{4}$ is a Poincaré ring etc. Finally, $E_{\infty}$ is a Poincaré ring. By Lemma 4 and (10) we get

$$
\tau(B) \tau(F)=\tau_{\xi_{2}}\left(E_{2}\right)=\tau_{\xi_{3}}\left(E_{3}\right)=\cdots=\tau_{\xi_{\infty}}\left(E_{\infty}\right),
$$

where $\xi_{r}$ (resp. $\xi_{\infty}$ ) is the image of $\xi_{2}$ in $E_{r}$ (resp. $E_{\infty}$ ).

It remains to prove that $\tau_{\xi_{\infty}}\left(E_{\infty}\right)=\tau(E)$. The cohomology ring $H^{*}(E)$ is filtered:

$$
\begin{gathered}
H^{*}(E)=D^{0} \supset D^{1} \supset \cdots \supset D^{p} \supset D^{p+1} \supset \cdots, \quad \cap D^{p}=0, \\
D^{p, q}=D^{p} \cap H^{p+q}(E), \\
D^{p, q} \cdot D^{p^{\prime}, q^{\prime}} \subset D^{p+p^{\prime}, q+q^{\prime}} .
\end{gathered}
$$

We have the filtration

$$
H^{r}(E)=D^{0, r} \supset D^{1, r-1} \supset \cdots \supset D^{r, 0} \supset D^{r+1,-1}=0
$$

and the canonical isomorphism

$$
D^{p, q} / D^{p+1, q-1} \cong E_{\infty}^{p, q} .
$$

The ring structure of $E_{\infty}$ is induced by that of $H^{*}(E)$ by the canonical homomorphisms $D^{p, q \rightarrow E_{\infty}^{p, q}}$ (see (12) and (11)). Since $E_{\infty}^{4 k}=E_{\infty}^{4 k-m, m}$, (where $m=\operatorname{dim} F$ ), we have

$$
H^{4 k}(E)=D^{4 k-m, m} \cong E_{\infty}^{4 k-m, m}
$$

and

$$
D^{4 k-i, i}=0
$$


Earlier we have chosen a generator $\xi_{\infty} \in E_{\infty}^{4 k}$. Under the canonical isomorphism (13) $\xi_{\infty}$ goes over in the generator $\xi_{E}$ of $H^{4 k}(E)$ belonging to the orientation of $E$ generated by the given orientations of $B$ and $F$ in this order. ${ }^{2}$ We now consider the bilinear symmetric function $\langle x, y\rangle$ over $H^{2 k}(E)$ relative to $\xi_{E}$. Choose a direct sum decomposition of $H^{2 k}(E)$ in linear subspaces,

$$
H^{2 k}(E)=V_{0}+V_{1}+V_{2}+\cdots+V_{m}
$$

such that

$$
\sum_{j=0}^{q} V_{j}=D^{2 k-q, q}
$$

Here we use that $D^{2 k-s, s}=D^{2 k-m, m}$ for $s>m$. By (11) and (14) we have

$$
\langle x, y\rangle=0 \quad \text { for } x \in V_{i}, y \in V_{i} \text { and } i+j<m,
$$

and moreover by (13)

$$
\langle x, y\rangle=\langle\tilde{x}, \tilde{y}\rangle, \quad \text { for } x \in V_{i}, y \in V_{j} \text { and } i+j=m,
$$

where $\tilde{x}$ (resp. $\tilde{y}$ ) denotes the image (see (12)) of $x$ (resp. $y$ ) in $E_{\infty}^{2 k-\imath,}$ (resp. $E_{\infty}^{2 k-j, J}$ ) and where on the right side of this equation stands the symmetric bilinear form over $E_{\infty}^{2 k}$ relative to $\xi_{\infty}$. Since $\langle\tilde{x}, \tilde{y}\rangle=0$ for $\tilde{x} \in E_{\infty}^{2 k-a, q}, \tilde{y} \in E_{\infty}^{2 k-q^{\prime}, \alpha^{\prime}}$, unless $q+q^{\prime}=m$, and since $E_{\infty}$ is a Poincaré algebra, we can conclude

$$
\operatorname{dim} E_{\infty}^{2 k-q, q}=\operatorname{dim} E_{\infty}^{2 k-m+q, m-q} .
$$

The preceding remarks, in particular (16), (17), (18), imply: The matrix of the symmetric bilinear function over $H^{2 k}(E)$ relative to $\xi_{B}$ can be written in blocks with respect to the direct sum decomposition (15) in the form

$$
\left(\begin{array}{cccc}
0 & & & L_{0} \\
& & L_{1} & \\
& \cdot & & \\
L_{m} & & & \\
& &
\end{array}\right)
$$

2 This is easy to see when $E$ is a trivial bundle, in which case it is almost the definition of the orientation of a product of manifolds. The general case can be reduced to this one by comparing the spectral sequence of $E$ to that of the bundle induced by $E$ on an open cell of the base, the cohomology being taken with compact carriers. 
where the $L_{i}$ are nonsingular square matrices and where $L_{i}$ is the transpose of $L_{m-i}$. Moreover,

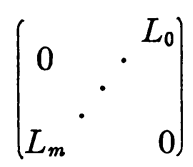

is the matrix of the symmetric bilinear function over $E_{\infty}^{2 k}$ relative to $\xi_{\infty}$. By Lemma 1 we have $\tau(E)=\tau_{\xi_{\infty}}\left(E_{\infty}\right)$. This concludes the proof of our theorem.

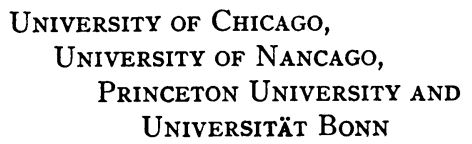

\section{THE PERIPHERAL CHARACTER OF CENTRAL ELEMENTS OF A LATTICE ${ }^{1}$}

\section{A. D. WALLACE}

A lattice being a Hausdorff space together with a pair of continuous lattice operations $(\wedge$ and $\vee$ ) the content of this note is best exhibited by quoting a corollary to our theorem: If a compact connected lattice is (topologically) situated in Euclidean n-space then its center is contained in its boundary. Thus, far from being "centrally located," the central elements are "peripheral."

The above is a consequence (see [3, p. 273]) of the

THEOREM. If $L$ is a compact connected lattice, if $R$ is an $(n, G)$-rim [3] for $L$ and if (i) $a$ is central [1, p. 27] or if (ii) $L$ is modular and $a$ is complemented then $a \in R$.

PROOF. The procedure is to introduce an appropriate multiplication into $L$ so that $L$ is a semigroup, to show that $L$ is not simple (in the semigroup sense [3]) and that $a$ is a left unit. Since $L$ is compact it has a zero and unit, 0 and 1 , as is well-known. Indeed, the set $\bigcap\{x \vee L \mid x \in L\}$ is easily seen to consist of exactly one element, namely 1 . If $a=1$ then the hypotheses of Theorem 1 of [3] are fulfilled using the multiplication $(x, y) \rightarrow x \wedge y$ so that 1 being a unit for the multiplication, $1 \in R$. If $a \neq 1$ let $x \cdot y=\left(a^{\prime} \wedge x\right) \vee y$, $a^{\prime}$ being a

Received by the editors September 15, 1956.

1 This work was supported by the National Science Foundation. 